\title{
Structural characterization of As-MIF and hJAB1 during the inhibition of cell-cycle regulation
}

\author{
Young-Hoon Park ${ }^{1, \#}$, Mi Suk Jeong ${ }^{1, \#}$, Ki-Tae $\mathrm{Ha}^{2}$, Hak Sun $\mathrm{Yu}^{3}$ E Se Bok Jang ${ }^{1, *}$ \\ ${ }^{1}$ Department of Molecular Biology, College of Natural Sciences, Pusan National University, Busan 46241, ${ }^{2}$ Department of Korean Medical \\ Science, School of Korean Medicine and Korean Medicine Research Centre for Healthy Aging, Pusan National University, Yangsan 50612, \\ ${ }^{3}$ Department of Parasitology, School of Medicine, Pusan National University, Yangsan 50612, Korea
}

\begin{abstract}
The biological activities of macrophage migration inhibitory factor (MIF) might be mediated through a classical receptormediated or non-classical endocytic pathway. JAB1 (C-Jun activation domain-binding protein-1) promotes the degradation of the tumor suppressor, p53, and the cyclin-dependent kinase inhibitor, p27. When MIF and JAB1 are bound to each other in various intracellular sites, MIF inhibits the positive regulatory effects of JAB1 on the activity of AP-1. The intestinal parasite, Anisakis simplex, has an immunomodulatory effect. The molecular mechanism of action of As-MIF and human JAB1 are poorly understood. In this study, As-MIF and hJAB1 were expressed and purified with high solubility. The structure of As-MIF and hJAB1 interaction was modeled by homology modeling based on the structure of Ace-MIF. This study provides evidence indicating that the MIF domain of As-MIF interacts directly with the MPN domain of hJAB1, and four structure-based mutants of As-MIF and hJAB1 disrupt the As-MIF-hJAB1 interaction. [BMB Reports 2017; 50(5): 269-274]
\end{abstract}

\section{INTRODUCTION}

Anisakis simplex is an intestinal nematode that causes human anisakidosis (1). This organism infects humans through the consumption of cephalopod species or raw marine fish containing its $3^{\text {rd }}$ stage larvae (2). When the $3^{\text {rd }}$ stage larvae migrate into the gastric mucosa, they cause abdominal pain, nausea, vomiting, and diarrhea (3). Some intestinal parasites, such as Anisakis simplex and Trichuris suis, have long life spans because they exert immunomodulatory effects (4). In

${ }^{*}$ Corresponding author. Tel: +82-51-510-2523; Fax: +82-51-5812544; E-mail: sbjang@pusan.ac.kr

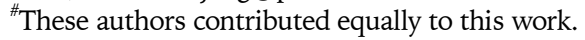

https://doi.org/10.5483/BMBRep.2017.50.5.201

Received 5 December 2016, Revised 19 December 2016, Accepted 29 March 2017

Keywords: As-MIF, Cell-cycle, hJAB1, Molecular interaction, Mutations particular, they regulate the host immune responses to resist protective mechanisms, and minimize severe pathological host changes (5). In previous studies several molecules from intestinal parasites down-regulated the host immune response. These molecules were identified as protease inhibitors, abundant larval transcript antigens, glycol-networks, venom allergen-like proteins, and mammal cytokine homologs (6).

A large-scale sequence analysis of Anisakis simplex $3^{\text {rd }}$ stage larvae was performed using the expressed sequence tags (ESTs) that isolated a MIF (macrophage migration inhibitory factor) homologue gene (7). In a mouse model of asthma, a recombinant MIF homolog from the larvae of the whale worm inhibited the Th2-type response, and reduced the levels of IL-4, -5, and -13 in the bronchial alveolar lavage fluid (BAL) (8, 9). In addition, an Anisakis simplex MIF (As-MIF)-treated group exhibited the complete inhibition of eosinophilia and goblet cell hyperplasia, and ameliorated the development of lung hyperreactivity (9). Although the enzymatic activities of MIF appear to require a membrane receptor-based mechanism of action, MIF also contains a tautomerase site with a catalytic site in the $\mathrm{N}$-terminal proline, which is consistent with the high conservation of this region across evolution (10). MIF is considered a potential target for use in the development of novel anti-inflammatory agents because of its membrane receptor-based cytokine action, telomerase activity and other intracellular functions (11).

Cell cycle regulation through an interaction with the co-activator and COP9 signalosome complex subunit 5 (CSN5), which is also known as the C-Jun activation domain-binding protein-1 (JAB1), was identified recently as an important intracellular function of MIF (12). MIF interacts with an intracellular protein, Jab1, which is a coactivator of AP-1 transcription (13). This study conducted a biochemical and biophysical analysis of As-MIF to reveal the interaction between As-MIF and hJAB1 in vitro. The results using protein models suggest that a complex of As-MIF and hJAB1 is formed by an interaction between the evolutionally conserved region of As-MIF and the MPN domain of hJAB1. Understanding the molecular interplay between As-MIF and JAB1-mediated signaling in vitro will help explain the complicated biological actions of As-MIF and hJAB1.

ISSN: 1976-670X (electronic edition)

Copyright (c) 2017 by the The Korean Society for Biochemistry and Molecular Biology

(c) This is an open-access article distributed under the terms of the Creative Commons Attribution Non-Commercial License (http://creativecommons.org/licenses/by-nc/4.0) which permits unrestricted non-commercial use, distribution, and reproduction in any medium, provided the original work is properly cited. 


\section{RESULTS AND DISCUSSION}

\section{Domain and secondary structures of As-MIF and hJAB1}

Fig. 1A presents the domain structures of full-length As-MIF (aa 1-121) and human JAB1 (1-334). The function of full-length As-MIF was not identified and As-MIF only has a macrophage migration inhibitory factor domain (MIF, 2-114). Full-length JAB1 is comprised of the Mpr1p Pad1p N-terminal domain (MPN, 53-138) and JAB1/MPN/Mov34 metalloenzyme (JAMM, 138-151) sequence motif located within the MPN domain. Three-dimensional modeling based on homology-modeling revealed the sequence of As-MIF to be most similar to that of Ancylostoma Ceylanicum (Ace-MIF) with a sequence identity of $36 \%$. The amino acid sequence of As-MIF was aligned with Ace-MIF (Fig. 1B). While the structure of Ace-MIF has seven $\alpha$-helices and six $\beta$-strands, the structure of As-MIF contains four $\alpha$-helices and five $\beta$-strands. The predicted secondary structure of As-MIF showed that three short $\alpha$-helices of Ace-MIF were changed to loops in As-MIF. The amino acids sequence of hJAB1 was aligned, as shown in Fig. 1C. MIF might mediate its biological activities through a classical receptor-mediated or non-classical endocytic pathway (13, 14). The pathways associated with MIF are involved in cell cycle regulation, transcription factors, and inflammation. JAB1 also promotes the degradation of the tumor suppressor, p53,

A

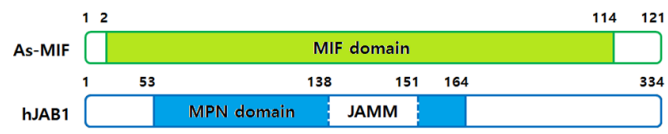

B

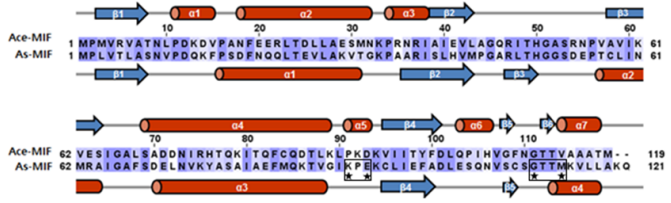

C

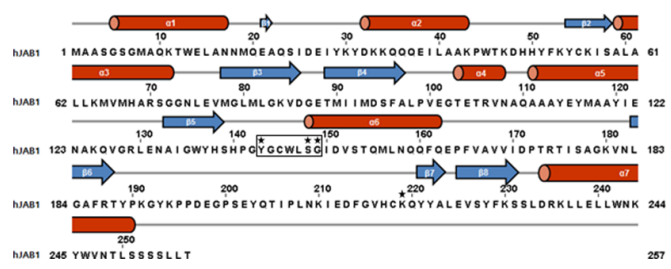

Fig. 1. (A) Schematic representation of the full-length As-MIF and hJAB1 domains. (B) Sequence alignment of As-MIF and Ace-MIF. The residues identically conserved in both species are in blue. The secondary structures of As-MIF and Ace-MIF were predicted. Alpha helices are shown as red cylinders, $\beta$-sheets as blue arrows, and loops as gray lines. The amino acids involved in the interactions with As-MIF and hJAB1 are indicated by stars. (C) The secondary structure of hJAB1 is shown. and the cyclin-dependent kinase inhibitor, p27. JAB1 interacts with MIF in the cytoplasm, where it functions as a coactivator of activator protein 1 (AP-1) transcription.

\section{Biochemical characterization of As-MIF and hJAB1}

Recombinant As-MIF and hJAB1 proteins were isolated and the soluble proteins were purified to homogeneity (Fig. 2A). An unsuccessful attempt was made to crystallize As-MIF and hJAB1 for a structural study. To investigate the oligomer structure of As-MIF, cross-linking was performed with $0.001 \%$ or $0.01 \%$ glutaraldehyde at $37^{\circ} \mathrm{C}$ (Fig. 2B). As shown in the wild-type As-MIF immunoblot, in addition to monomeric species including His-tag $(17 \mathrm{kDa})$, protein bands were observed at high molecular weights corresponding to dimeric and homotrimeric structures in the presence of all non-reducing As-MIF (No boiling, 0.001 and $0.01 \%$ ). To further investigate the nature of the secondary structural elements of the purified full-length As-MIF and hJAB1 proteins, far-UV CD spectra were recorded (Fig. 2C, D). The CD spectra
A

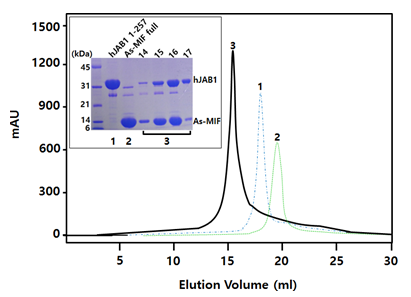

C
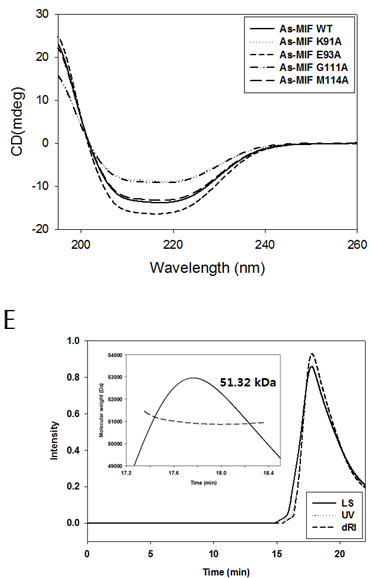

B

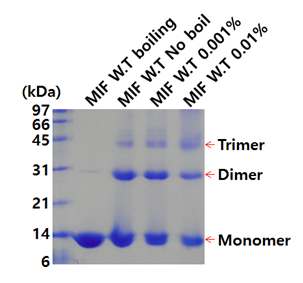

$\mathrm{D}$

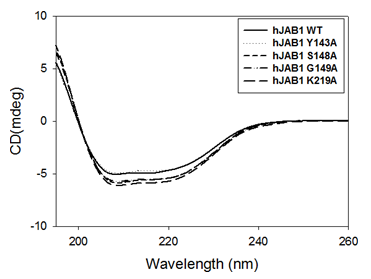

Fig. 2. (A) After the application of SEC, the interactions between As-MIF and hJAB1 (1-257) were revealed. (B) Cross-linking analysis of wild-type As-MIF using $0.001 \%$ and $0.01 \%$ glutaraldehyde in HEPES (pH 8.0) buffer. (C, D) Far-UV CD spectra of As-MIF/hJAB1 and their mutants are shown. (E) MALS analysis of the wild-type As-MIF is shown. MALS, dRI, and UV spectra for calculating the molecular mass of the As-MIF are shown. 
of the As-MIF and hJAB1 were calculated using CDNN and they were presumed to have predominantly $\alpha$-helical structures.

JAB1 has a JAMM metalloenzyme sequence motif located within the MPN domain. Incubation with the recombinant and vesicular MIF produced a significant decrease $(\sim 50 \%)$ in the zinc content in stimulated caput sperm (15). Inductively coupled plasma-mass spectrometry (ICP-MS) was performed to determine if zinc binds to As-MIF or hJAB1. The $\mathrm{Zn}$ ion concentration was $205 \mathrm{ppb}$ and $435 \mathrm{ppb}$ for As-MIF and hJAB1, respectively. Zinc can be bound to cysteines, and the cysteine mutants (C95A and C109A) showed inferior Zn binding ability (220 and $165 \mathrm{ppb}$ ) to that of wild-type MIF. In addition, the cysteine mutants (C145A and C218A) also showed inferior $\mathrm{Zn}$ binding ability (296 and $177 \mathrm{ppb}$ ) to that of wild-type hJAB1. The molecular weight of the full-length As-MIF was characterized by measuring the LS, dRI, and UV spectra by size exclusion chromatography-MALS analysis (Fig. $2 \mathrm{E})$. The weight-average molecular weight of the As-MIF solution was $51 \mathrm{kDa}$, corresponding to the size of the full-length As-MIF trimer.

\section{Structural prediction of As-MIF and hJAB1 interaction}

An accurate prediction of the protein-protein interactions is an important step toward elucidating the protein function and
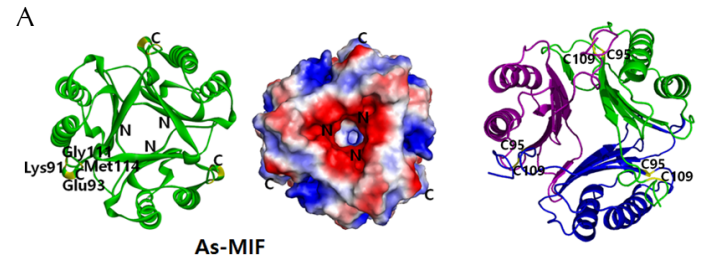

B

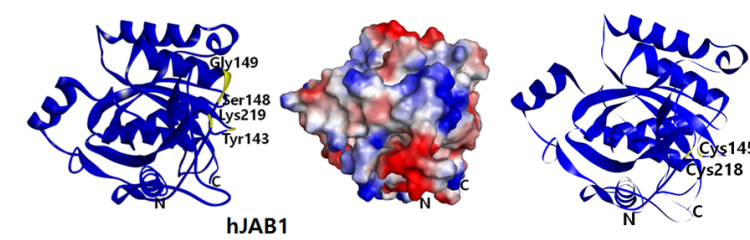

C
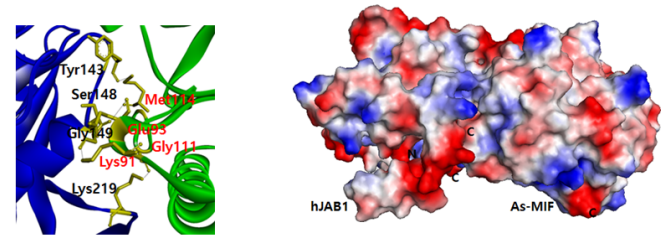

Fig. 3. (A) Three-dimensional structure of As-MIF (2-117, green) is shown as ribbon (left) and surface representations (middle). The cysteine residues (C95 and C109) of As-MIF are shown (right). (B) Three-dimensional structure of hJAB1 (2-257, blue) is shown. (C) The complex structure of As-MIF and hJAB1 was modeled in ribbon (left) and surface representations (right). The binding sites (yellow) between As-MIF and hJAB1 are illustrated. understanding of the molecular mechanisms inside the cell. To investigate the structural aspects of As-MIF and hJAB1 interaction, the As-MIF (2-116) structure was modeled using the known structure of Ace-MIF (PDB ID: 2OS5) (Fig. 3A). The hJAB1 (PDB ID: 4F7O, 2-256) was used for interaction modeling (Fig. 3B). To accomplish interaction modeling between As-MIF and hJAB1, ten predicted low energy structures formed in 1,000 docking run structures were selected. This study focused on one model of the ten complexes for further analysis. The As-MIF-hJAB1 complex model was analyzed by Ramachandran plot calculations and peptide torsion $\omega$ angles using a validation tool $(16,17)$. The validation analysis indicated that predicted model showed the adequate stereochemistry, with almost $100 \%$ of the amino acid side chains located in allowed region of a $[\varphi, \psi]$ plot.

The C-terminal in the MIF domain of As-MIF was predicted to dock into the MPN domain of hJAB1. The important binding sites of As-MIF and hJAB1 were identified using the modeled complex structures (Fig. 3C). Four binding residues of As-MIF were selected for mutation to examine the interactions between As-MIF and hJAB1. Four residues on the loop (K91, E93, G111, and M114) of As-MIF and four on the loop (Y143, S148, G149, and K219) of the hJAB1 regions may be important interaction residues between As-MIF and hJAB1. K91, E93, G111 and M114 of As-MIF may hydrogen bond with G149, S148, K219 and Y143 of the hJAB1, respectively.
A

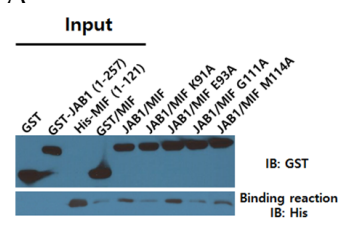

C

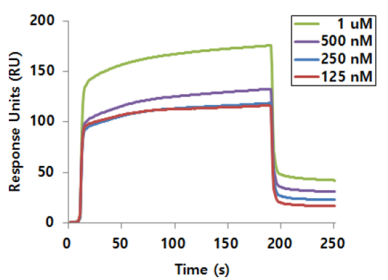

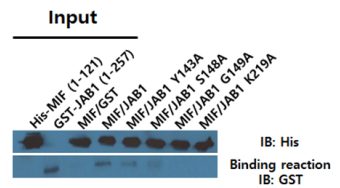

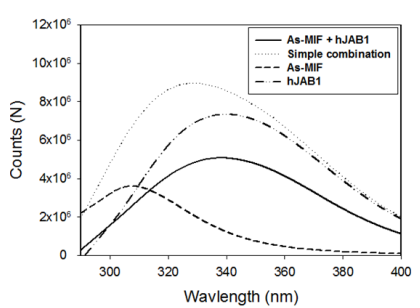

Fig. 4. (A) Binding analysis between the structure-based mutations (K91A, E93A, G111A and M114A) of As-MIF and hJAB1 was conducted by a GST-tag pull-down assay in vitro. (B) Binding analysis of As-MIF and structure-based mutations (Y143A, S148A, G149A, and K219A) of hJAB1 are shown by a His-tag pull-down assay. The protein interactions were detected by Western blot. (C) Biacore biosensor analysis of As-MIF and hJAB1 is shown. The As-MIF binds to hJAB1. (D) Fluorescence spectra of the As-MIF-hJAB1 complex and each individual protein are shown. 
K91 of As-MIF and K219 of hJAB are positively charged residues, and these charges may promote the formation of the As-MIF-JAB1 complex. The As-MIF trimer contains a hole surrounded by negatively charged residues. The negatively charged surface of As-MIF interacts with the positively charged surface of hJAB1; these interactions are localized in the loop regions (Fig. 3). Protein loop regions are often flexible and frequently adopt several conformations.

Interestingly, the $\mathrm{K} 91 \mathrm{~A}$ and $\mathrm{G} 111 \mathrm{~A}$ mutations in As-MIF disrupted their interactions with hJAB1 (Fig. 4A). These two mutations destabilized As-MIF structurally. Of the four mutations produced by the substitution of Ala in the hJAB1, two, G149A and K219A on hJAB1, were unable to participate in the As-MIF and JAB1 interactions (Fig. 4B). These results suggest that the predicted interaction sites in the modeled complex structures play important roles in the structural stability of the As-MIF and hJAB1 protein complex. The loop region of As-MIF is docked to the MPN domain, including the JAMM motif of the hJAB1 structure. Recently, the crystal structure of an eight-subunit COP9 signalosome complex (CSN) was determined $(18,19)$. CSN has two organizational centers: a horseshoe-shaped ring formed by its six proteasome lid-CSN-initiation factor $3(\mathrm{PCl})$ domain proteins, and a large bundle formed by the carboxy-terminal $\alpha$-helices of every subunit. In the structure, an important component of the COP9 signalosome complex JAB1 (CSN5) and its dimerization partner, CSN6, are embedded intricately at the core of the helical bundle.

\section{Binding between As-MIF and JAB1 in vitro}

A series of biochemical and biophysical experiments were conducted to determine if As-MIF interacts with hJAB1 in vitro. The interactions were identified using a size-exclusion column (Fig. 2A). Binding between As-MIF and hJAB1 was detected using SDS-PAGE. The complex of As-MIF and hJAB1 was shown as a sharp peak. The complex was eluted earlier than each of the protein peaks, indicating that As-MIF interacts with hJAB1 in vitro. The use of the His-tagged As-MIF fusion protein was effective in the pull-down of GST-hJAB1 (Fig. 4A). Binding between As-MIF and hJAB1 was detected by Western blot analysis. In addition, the GST-hJAB1 fusion protein was used in the pull-down of His-tagged As-MIF. Two mutants of As-MIF (K91A, and G111A) did not interact with hJAB1. In the case of the hJAB1 mutants, G149A and K219A did not interact with As-MIF (Fig. 4B). Biacore biosensor analysis was conducted to measure the JAB1-binding ability between the full-length As-MIF and hJAB1 (Fig. 4C). Sensorgrams of As-MIF binding to hJAB1 were used to calculate their kinetic binding constants. Then the background sensorgrams were substracted from the experimental sensorgrams, yielding representative specific binding constants. The As-MIF was physically bound to hJAB1 with an apparent $K_{D}$ of $16.56 \mathrm{nM}$ (Supplementary Table 1).

To further investigate the interaction between As-MIF and hJAB1, their fluorescence emission spectra were measured with a $\lambda_{\max }$ at $310 \mathrm{~nm}$ or $340 \mathrm{~nm}$, respectively (Fig. 4D). The fluorescence intensities of As-MIF, hJAB1, and their complex were approximately $3.5 \times 10^{6} \mathrm{~N}, 7.5 \times 10^{6} \mathrm{~N}$, and $5.0 \times 10^{6}$ $\mathrm{N}$, respectively. The fluorescence intensity of hJAB1 increased significantly in response to the exposed tryptophan residue, suggesting that the Trp fluorescence of hJAB1 originates primarily from the aromatic residues. Although hJAB1 has six Trp amino acids, As-MIF has none, and its fluorescence emission spectrum was shifted slightly to a lower wavelength because of the emission of Phe. The spectrum of the As-MIF and hJAB1 complex showed lower intensity than that observed when simply combining As-MIF and hJAB1. Large conformational changes in response to their interaction occurred in one or both proteins and the residues of the aromatic groups were buried within the protein structures.

In this study, the As-MIF and hJAB1 proteins produced by recombinant $E$. coli were purified and characterized. A series of biochemical and biophysical measurements confirmed that As-MIF interacts with hJAB1 in vitro. Interestingly, two mutations (K91A and G111A) of As-MIF and two mutations (G149A and K219A) of hJAB1 disrupted the interaction between As-MIF and hJAB1. The results provide important data regarding the structures of As-MIF and hJAB1, the binding activity of As-MIF to hJAB1, and the protein-protein interactions involved in cell cycle regulation, transcription factors, and inflammation. Overall, an improved understanding of the molecular interaction between As-MIF and hJAB1 will help elucidate the biological actions of As-MIF and hJAB1.

\section{MATERIALS AND METHODS}

\section{Cloning and mutation of As-MIF and hJAB1}

The full-length As-MIF (1-121) was subcloned in the $\mathrm{N}$-terminal of the His-tagged fusion protein vector, $\mathrm{pET}-26 \mathrm{~b}$. The hJAB1 (1-257) sequence was subcloned into another His-tagged fusion protein vector, pET28a. As-MIF and hJAB1 were amplified by a polymerase chain reaction (PCR) using the oligonucleotides incorporating the $\mathrm{Ndel} / \mathrm{Xhol}$ and $\mathrm{BamHI} / \mathrm{Xhol}$ sites on the $5^{\prime}$ and $3^{\prime}$ primers, respectively. Double-stranded oligonucleotides were used for site-directed mutagenesis of six different As-MIF residues to alanine (K91A, E93A, G111A, M114A, C95A, and C109A). Site-directed mutagenesis of the four different hJAB1 residues to alanine (Y143A, S148A, G149A and K219A) was performed. All cloned cDNAs were identified by restriction endonuclease digestion and verified using a Macrogen automatic DNA sequencer.

\section{Expression and purification of recombinant protein}

The His-tagged As-MIF and hJAB1 were transformed to the competent cell, Escherichia coli BL21(DE3), which contains many T7 polymerases. Each colony was inoculated overnight at $37^{\circ} \mathrm{C}$ in $5 \mathrm{ml}$ of Luria Bertani (LB) medium enriched with 10 $\mu \mathrm{g} / \mathrm{ml}$ kanamycin. The cells were incubated in LB containing kanamycin and maintained at $37^{\circ} \mathrm{C}$ until the $\mathrm{OD}_{600}$ reached 
0.5-0.6. Protein expression was induced by $0.5 \mathrm{mM}$ isopropyl-thio- $\beta$-D-galactopyranoside (IPTG) overnight at $25^{\circ} \mathrm{C}$, after which the bacterial cells were harvested by centrifugation at $3,660 \mathrm{~g}$ for $25 \mathrm{~min}$ at $4^{\circ} \mathrm{C}$. The cell pellets were used immediately or frozen at $-70^{\circ} \mathrm{C}$, then re-suspended with lysis buffer A [50 mM Tris- $\mathrm{HCl}(\mathrm{pH} 8.0)$ and $200 \mathrm{mM} \mathrm{NaCl}]$ and sonicated for cell disruption. After sonication, the cell suspensions were centrifuged at 20,170 g for $45 \mathrm{~min}$ to remove the insoluble cellular debris. The soluble supernatants of the His-tagged As-MIF and hJAB1 were loaded onto a Ni-NTA column and pre-equilibrated with buffer $A$, after which the bound proteins were eluted using buffer A containing 20-200 mM imidazole. The final volume of the eluted supernatants was concentrated using a Vivaspin 20 at 1,320 g. The concentration fractions of As-MIF and hJAB1 from the Ni-NTA column were purified by gel filtration chromatography using a Superdex 200 10/300 GL fast protein liquid chromatography (FPLC) column (Amersham-Pharmacia Biotech) equilibrated in buffer A. Purification step was analyzed by $15 \%$ SDS-PAGE gel and visualized by Coomassie blue staining.

\section{Circular dichroism spectroscopy analysis}

The circular dichroism (CD) spectra of full-length As-MIF, hJAB1, and their mutants were measured on a JASCO J-715 spectropolarimeter in a $0.1 \mathrm{~cm}$ cell at $25^{\circ} \mathrm{C}$. The CD spectra of the purified proteins $(0.5 \mathrm{mg} / \mathrm{ml})$ were recorded in the $190-260$ $\mathrm{nm}$ range. The secondary-structural elements were calculated using the CDNN program.

\section{Fluorescence spectroscopy}

The fluorescence emission spectra were obtained using an Edinburgh (UK) FLS920 TCSPC (Time Correlated Single Photon Counting Spectrometer) with $1 \mathrm{~cm}$ pathlength cuvettes containing excitation and emission slits, $20 \mathrm{~nm}$ in width. The fluorescence emission spectra of As-MIF and hJAB1 were obtained to identify the characteristic chemical structures, namely double bonds and aromatic groups. The emission intensity was recorded from 280 to $400 \mathrm{~nm}$ at an excitation wavelength of $295 \mathrm{~nm}$. As-MIF and hJAB1 were pre-incubated for $25 \mathrm{~min}$ at $25^{\circ} \mathrm{C}$.

\section{Glutaraldehyde cross-linking experiment}

Cross-linking was performed with $10 \mu \mathrm{g}$ of the interacting proteins at various concentrations. Wild-type As-MIF and each mutant in a $20 \mathrm{mM}$ HEPES reaction buffer $(\mathrm{pH}$ 8.0) were treated with $0.001 \%$ or $0.01 \%$ glutaraldehyde for $2 \mathrm{~min}$ at $37^{\circ} \mathrm{C}$. The reaction was quenched by the addition of $1 \mathrm{M}$ Tris- $\mathrm{HCl}(\mathrm{pH}$ 7.0) for $10 \mathrm{~min}$ at room temperature.

\section{Multi-angle light scattering (MALS)}

Molecular weights (MW), differential refractive index spectra $(\mathrm{dRI})$, and ultraviolet (UV) spectra were measured using a MALS and dRI (Wyatt Technology Corporation) detector coupled with a high-performance liquid chromatograph (Shimadzu, Kyoto, Japan). The purified full-length As-MIF was loaded onto a size-exclusion column that had been preequilibrated with the standard buffer at a flow rate of 0.5 $\mathrm{ml} / \mathrm{min}$. The molecular weight was calculated using ASTRA 6.0 (Wyatt Technology Corporation).

\section{Inductively Coupled Plasma-Mass Spectrometry (ICP-MS)}

Inductively coupled plasma-mass spectrometry (ICP-MS) was performed using an Elan 6100 DRC ICP-MS instrument (Perkin Elmer Life and Analytical Sciences, Shelton, CT, USA). The plasma gas flow rate, radio frequency, and radio power were $15 \mathrm{~L} \cdot \min ^{-1}, 40 \mathrm{MHz}$, and $1.0 \mathrm{~kW}$, respectively. Metal analysis for $\mathrm{Zn}^{2+}$ (M.W. $65.39 \mathrm{~g} / \mathrm{mol}$ ) was performed by combustion analysis using a FLASH EA 1112 elemental analyzer (Thermo Electron Corporation). The protein samples were dried at $50^{\circ} \mathrm{C}$ for $12 \mathrm{~h}$ in a vacuum prior to metal analysis.

\section{Structural modeling}

As-MIF (2-116) and hJAB1 (2-257) models were constructed using SWISS-MODEL software, which is a relative threedimensional protein modeling system. The results of an Expert Protein Analysis System (EXPASY) search of the protein data bank (PDB) revealed a template protein with considerable sequence identity. 3D models of As-MIF and hJAB1 were prepared using the homology protein templates of Ancylostoma ceylanicum MIF (Ace-MIF; PDB ID: 2OS5) and COP9 signalosome complex subunit 5 (PDB ID: 4F7O). As-MIF (2-116) and hJAB1 (2-257) were bound and the most stable complex structure was selected from the top 20 complexes obtained from each docking.

\section{GST pull-down assay}

For the pull-down assay, purified GST, GST-JAB1, and GST-As-MIF were mixed with His-tagged MIF, hJAB1 and their mutants, which were incubated with buffer $A$ and pre-equilibrated glutathione sepharose $4 \mathrm{~B}$ beads. After a $4 \mathrm{~h}$ reaction at $4^{\circ} \mathrm{C}$, the beads were centrifuged at 1,320 g for 4 min, and washed with buffer $A$. For each time, the supernatant was removed by centrifuging. The binding proteins were eluted with the buffer $[50 \mathrm{mM}$ Tris- $\mathrm{HCl}(\mathrm{pH} \mathrm{8.0)}$ and $30 \mathrm{mM}$ glutathione]. The proteins were visualized by $15 \%$ SDS gel and Western blot.

\section{His pull-down assay}

Purified His-tagged As-MIF was mixed with GST-tagged JAB1 and their mutants, which were incubated with buffer $A$ and pre-equilibrated Ni-NTA agarose beads. After an $8 \mathrm{~h}$ reaction at $4^{\circ} \mathrm{C}$, the beads were centrifuged and washed with buffer $\mathrm{A}$. The supernatant was removed by centrifuging at 1,320 $\mathrm{g}$ for 2 min. The binding proteins were eluted with the buffer $A$ with 1 $M$ imidazole. 


\section{Biacore biosensor analysis}

Measurements of the apparent dissociation constants $\left(\mathrm{K}_{\mathrm{D}}\right)$ between As-MIF and hJAB1 were carried out using a Biacore T100 biosensor (GE Healthcare Biosciences, Sweden). The purified As-MIF was bound covalently to the sensor chip CM5 (carboxylated dextran matrix) using an amine-coupling method. The As-MIF $(40 \mu \mathrm{g} / \mathrm{ml})$ in $10 \mathrm{mM}$ sodium acetate $\mathrm{pH}$ 5.0 was coupled via injection for $15 \mathrm{~min}$ at $10 \mu \mathrm{l} / \mathrm{min}$, followed by the injection of $1 \mathrm{M}$ ethanolamine to deactivate residual amines. For kinetic measurements at $25^{\circ} \mathrm{C}, \mathrm{hJ} A \mathrm{AB} 1$ with concentrations ranging from 125 to $1,000 \mathrm{nM}$ was prepared by dilution in $\mathrm{HBS}^{-\mathrm{EP}^{+}}$buffer $(10 \mathrm{mM}$ of HEPES, $\mathrm{pH}$ $7.4,150 \mathrm{mM}$ of $\mathrm{NaCl}, 3 \mathrm{mM}$ of EDTA and $0.05 \% \mathrm{v} / \mathrm{v}$ surfactant P20). Immobilized ligand was regenerated by injecting of $50 \mathrm{mM} \mathrm{NaOH}$ at a rate $10 \mu \mathrm{l} / \mathrm{min}$ during the cycles.

\section{ACKNOWLEDGEMENTS}

This study was supported by the Basic Science Research Program through the National Research Foundation of Korea (NRF) funded by the Ministry of Education, Science and Technology (2015R1D1A1A01059594) to S.B.J. and (2016R 1D1A1B02011142) to J.M.S.

\section{CONFLICTS OF INTEREST}

The authors have no conflicting financial interests.

\section{REFERENCES}

1. Bouree P, Paugam A and Petithory JC (1995) Anisakidosis: report of 25 cases and review of the literature. Comp Immunol Microb 18, 75-84

2. Hochberg NS, Hamer DH, Hughes JM and Wilson ME (2010) Anisakidosis: perils of the deep. Clin Infect Dis 51, 806-812

3. Deardorff TL, Fukumura T and Raybourne RB (1986) Invasive anisakiasis: a case report from Hawaii. Gastroenterology 90, 1047-1050

4. Maizels RM and Yazdanbakhsh M (2003) Immune regulation by helminth parasites: cellular and molecular mechanisms. Nat Rev Immunol 3, 733-744

5. Weinstock JV, Summers R and Elliott DE (2004) Helminths and harmony. Gut 53, 7-9

6. Thomas PG, Carter MR, Harn DA et al (2003) Maturation of dendritic cell 2 phenotype by a helminth glycan uses a Toll-like receptor 4-dependent mechanism. J Immunol 171, 5837-5841

7. Yu HS, Park SK, Jeong HJ et al (2007) Anisakis simplex: analysis of expressed sequence tags (ESTs) of third-stage larva. Exp Parasitol 117, 51-56

8. Zang X, Taylor P, Maizels RM et al (2002) Homologues of human macrophage migration inhibitory factor from a parasitic nematode. Gene cloning, protein activity, and crystal structure. J Biol Chem 277, 44261-44267

9. Park SK, Cho MK, Yu HS et al (2009) Macrophage migration inhibitory factor homologs of anisakis simplex suppress Th2 response in allergic airway inflammation model via CD4 + CD25 + Foxp3 + T cell recruitment. J Immunol 182, 6907-6914

10. Dobson SE, Augustijn KD, Wilkinson AJ et al (2009) The crystal structures of macrophage migration inhibitory factor from Plasmodium falciparum and Plasmodium berghei. Protein Sci 18, 2578-2591

11. Lubetsky JB, Dios A, Al-Abed Y et al (2002) The tautomerase active site of macrophage migration inhibitory factor is a potential target for discovery of novel anti-inflammatory agents. J Biol Chem 277, 24976-24982

12. Kleemann R, Hausser A, Bernhagen J et al (2000) Intracellular action of the cytokine MIF to modulate AP-1 activity and the cell cycle through Jab1. Nature 408, 211-216

13. Claret FX, Hibi M and Dhut S (1996) A new group of conserved coactivators that increase the specificity of AP-1 transcription factors. Nature 383, 453-457

14. Bianchi E, Denti S and Granata A (2000) Integrin LFA-1 interacts with the transcriptional co-activator JAB1 to modulate AP-1 activity. Nature 404, 617-621

15. Eickhoff R, Baldauf C, Koyro HW et al (2004) Influence of macrophage migration inhibitory factor (MIF) on the zinc content and redox state of protein-bound sulphydryl groups in rat sperm: indications for a new role of MIF in sperm maturation. Mol Hum Reprod 605, 605-611

16. Lovell SC, Davis IW, Arendall WB, de Bakker PI, Word JM and Richardson DC (2003) Structure validation by $C \alpha$ geometry: $\phi, \psi$ and $C \beta$ deviation. Proteins $50,437-450$

17. Emsley P, Lohkamp B, Scott WG and Cowtan K (2010). Features and development of Coot. Acta Crystallogr D 66, 486-501

18. Lingaraju GM, Bunker RD, Cavadini S et al (2014) Crystal structure of the human COP9 signalosome. Nature 512, 161-165

19. Cavadini S, Fischer ES, Bunker RD et al (2016) Cullin-RING ubiquitin E3 ligase regulation by the COP9 signalosome. Nature 531, 598-603 